\title{
Endocrine Manifestations of Langerhans Cell Histiocytosis
}

Cardoso L1, Gomes L1,2, Rodrigues $D^{1,2}$, Gouveia $\mathbf{S}^{1,2}$, Saraiva $\mathrm{J}^{1,2}$, Moreno $\mathbf{C}^{1}$, Guelho $\mathrm{D}^{1}$, Dantas $\mathbf{R}^{3}$, Vicente $\mathbf{N}^{1}$, Balsa $\mathbf{M}^{3}$, Martins $\mathrm{D}^{1}$, Oliveira $\mathrm{D}^{1}$, Carrilho F1 Department of Endocrinology, Diabetes and Metabolism, Centro Hospitalar e Universitário de Coimbra, Coimbra, Portugal 2. Faculty of Medicine, University of Coimbra, Coimbra, Portugal

3. Department of Endocrinology, Centro Hospitalar do Baixo Vouga, Aveiro, Portugal

\section{Introduction}

Langerhans cell histiocytosis $(\mathrm{LCH})$ is a rare granulomatous and proliferative disease. Clinical presentation and aggressiveness are very heterogeneous, extending from very benign to disseminated forms, that causes significant mortality. Indeed, LCH aggregates in one entity a group of diseases characterized by clonal proliferation and/or accumulation of cells phenotypically similar to cutaneous Langerhans cells (surface markers and ultrastructure). Multisystem forms frequently have hypothalamo-pituitary axis involvement.

\section{Clinical Case 1}

A 15-year-old Caucasian woman presented with a 14-month history of headaches, secondary amenorrhea. She reported polyuria and polydipsia in the last 2 years.
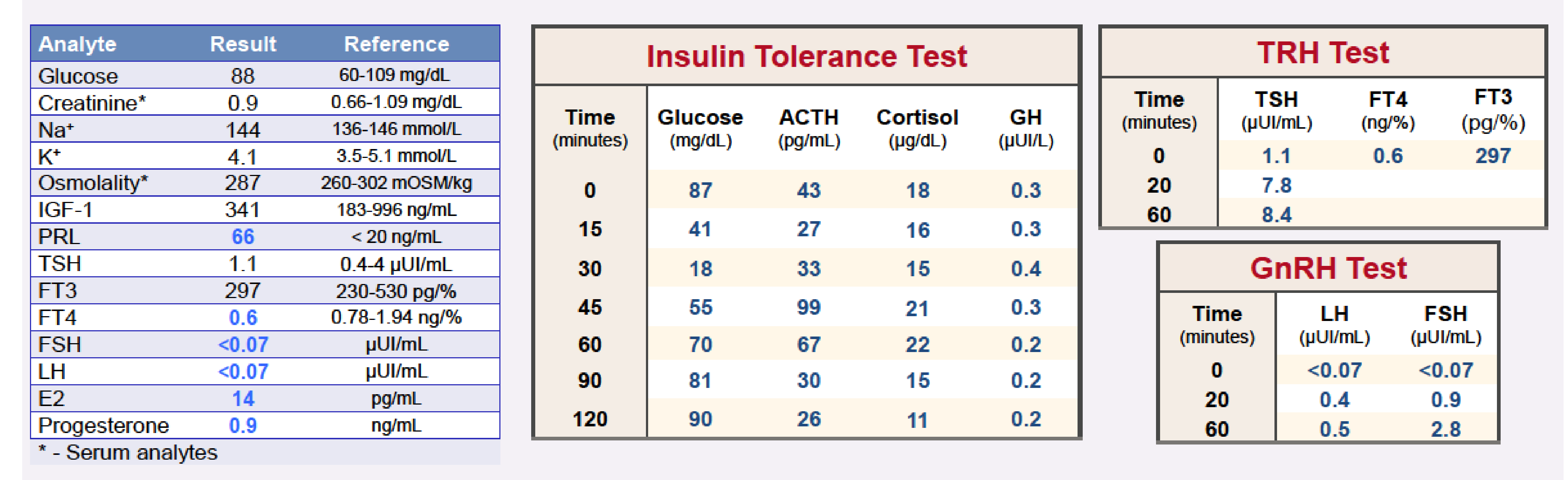

Imaging: hypothalamic mass of $10 \mathrm{~mm}$
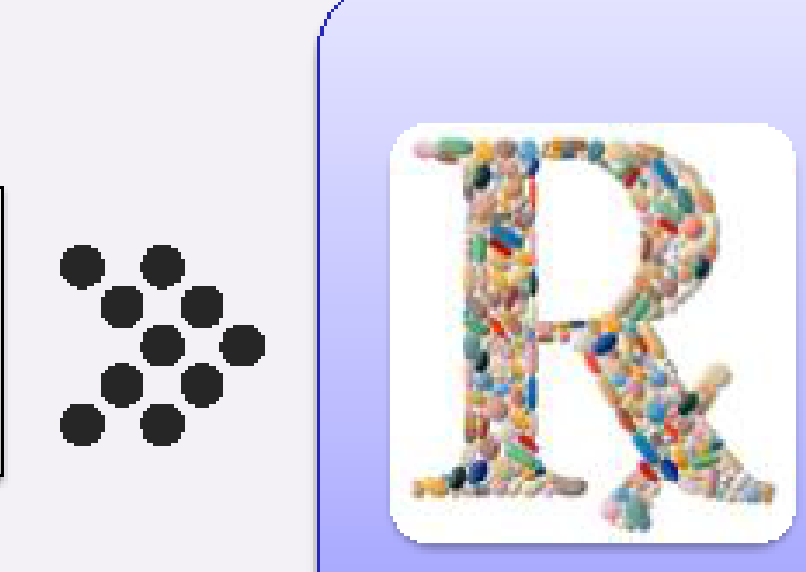

Pituitary transcranial surgery After surgery: panhypopituitarism

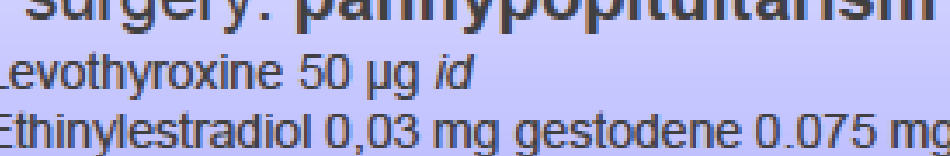
Histology: inconclusive

\section{9 monts after surgery.}

MRI: hypothalamic residue of $14 \times 12.8 \mathrm{~mm}$

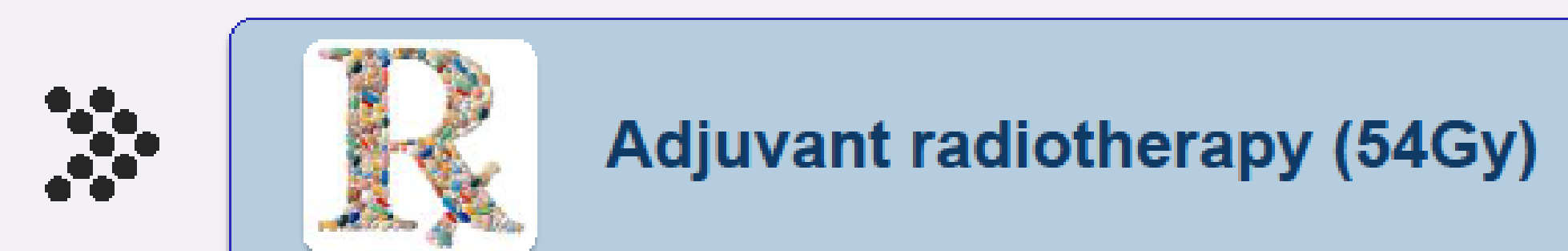

\section{3 years after surgery}

Patient reported a 16-month history of vulvar ulceration, to which she had been submitted to three surgical excision procedures in the previous year.

\section{Vulvar Histology: Langerhans Cell Histiocytosis}
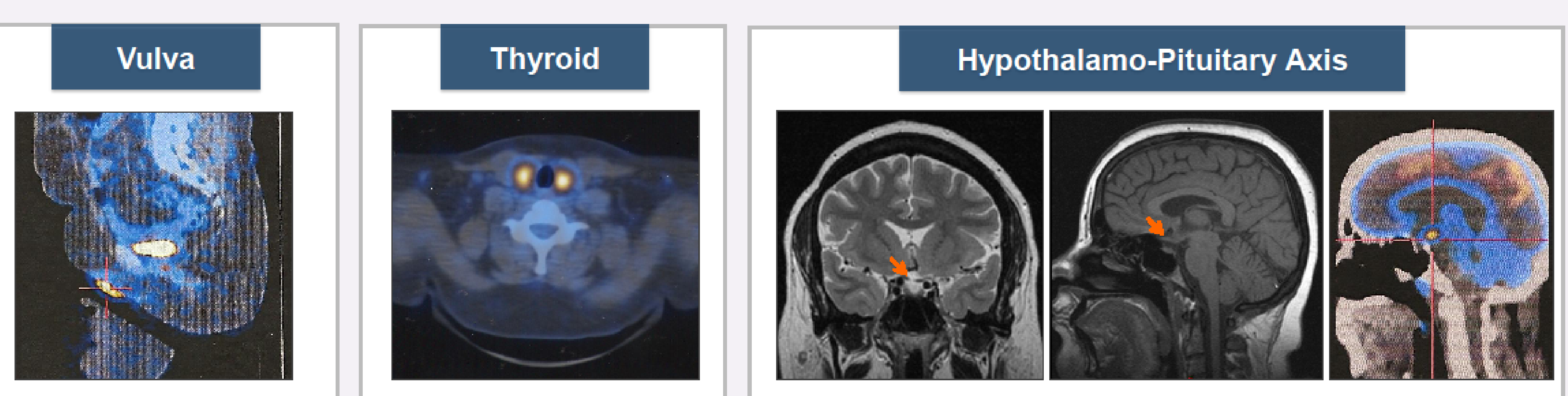

\section{Clinical Case 2}

A 37-year-old Caucasian man presented with a 6-month history of otalgy, otorrhea, weight loss, and brown maculo-papular skin rash. He reported polyuria, and polydipsia in the previous 5 years. Recently he noted vision impairment. A mass in the temporal bone was found. Imaging studies revealed osteolytic lesions in the temporal bone and a $2 \mathrm{~cm}$ suprasellar mass.
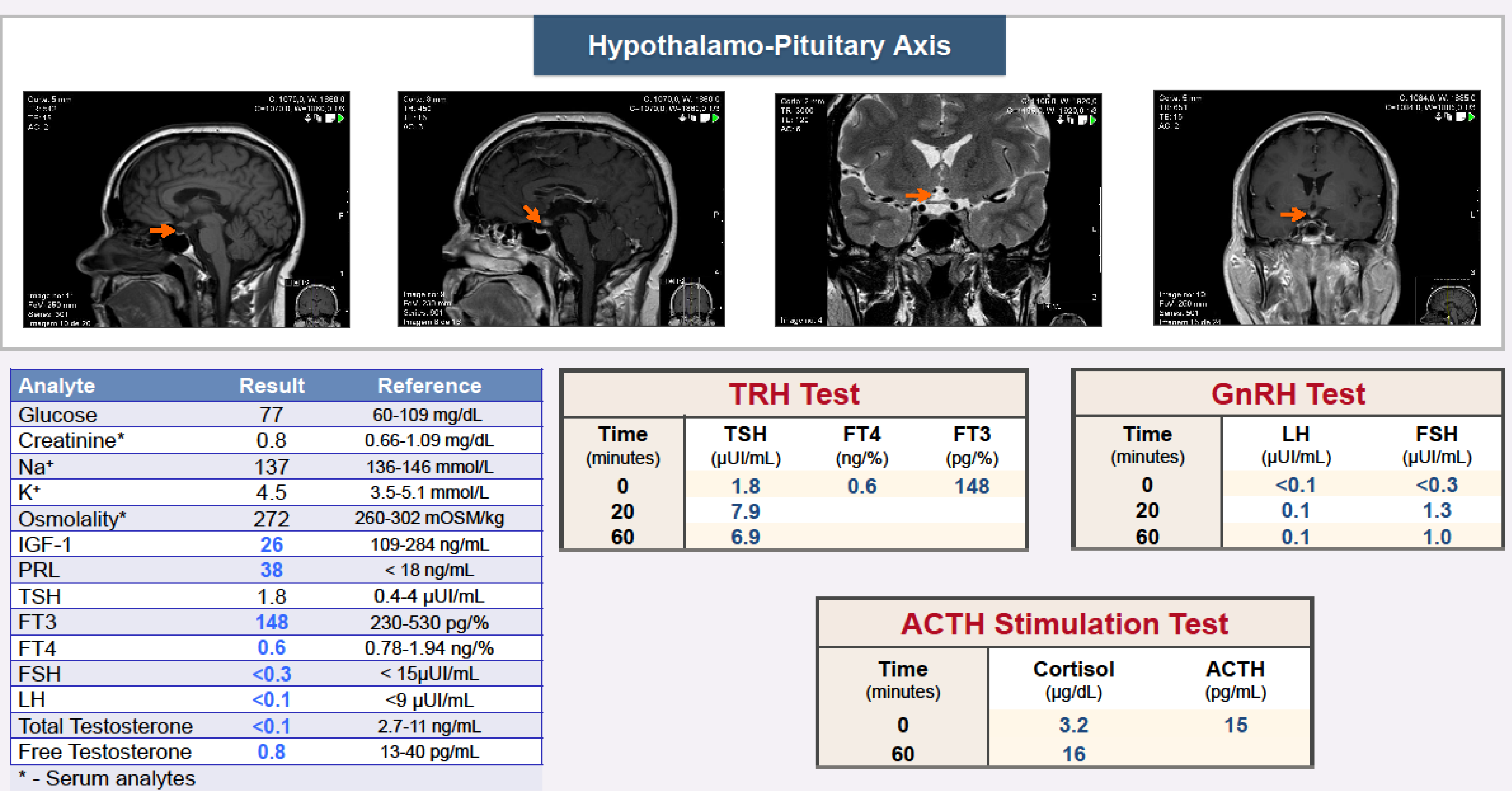

Temporal Bone Histology: Langerhans Cell Histiocytosis

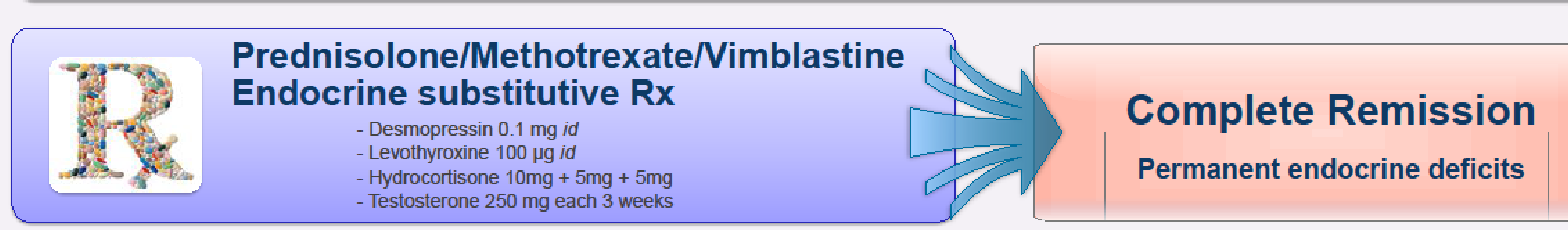

\section{Clinical Case 3}

A 60-year-old Caucasian woman presented with 6-month history of jaw pain and weight loss. Secondary evaluation revealed polyostotic Langerhans cell histiocytosis and the patient was treated with zoledronic acid. Three years later she complained of headaches, polyuria and polydipsia. MRI revealed pituitary stalk enlargement.

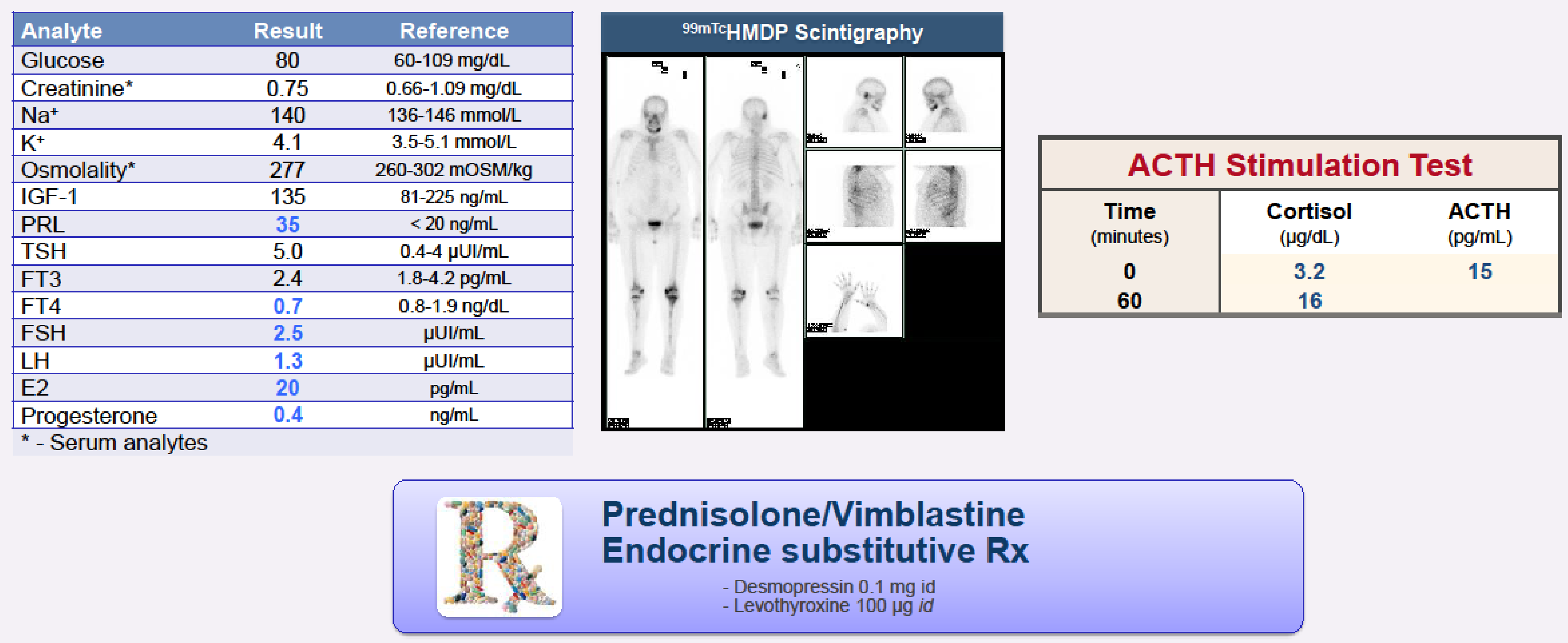

\section{Conclusion}

- All cases described had hypothalamo-pituitary axis involvement with pituitary failure. The dominant manifestation was diabetes insipidus.

- If not treated promptly LCH may have a considerable morbi-mortality with permanent endocrine failures, that require proper hormonal substitution. Relapses may occur and appropriate follow-up is recommended. 